\title{
Modernisierung der nass betriebenen Aufbereitungsanlage im Werk Aspang
}

\author{
Georg Partlic ${ }^{1}$ und Helfried Gschaider ${ }^{2}$ \\ 'Lehrstuhl für Rohstoffmineralogie, Montanuniversität Leoben, Leoben, Österreich \\ ${ }^{2}$ Binder+Co AG, Gleisdorf, Österreich
}

Eingegangen 4. Juni 2018; angenommen 13. Juni 2018; online publiziert 16. Juli 2018

\begin{abstract}
Zusammenfassung: Die nass betriebene Aufbereitungsanlage der Aspanger Bergbau und Mineralwerke $\mathrm{GmbH} \& \mathrm{Co}$ KG dient zur Aufbereitung von Muskovit-Glimmer aus dem im Tagbau gewonnenen Leukophyllit. Die Modernisierung der Anlage hatte als Zielsetzung, einerseits das Ausbringen an Muskovit-Glimmer zu erhöhen, andererseits die Energieeffizienz zu steigern, wobei die Steigerung des Ausbringens durch die Installation einer Attritionsmaschine erzielt wird. Zusätzlich wurde die Aufbereitungsanlage dahingehend adaptiert, dass in einem zweiten Produktionsweg gewaschener Quarzsand für die Betonindustrie aus den überlagernden Semmeringquarziten produziert werden kann.
\end{abstract}

Schlüsselwörter: Modernisierung, Aspanger, Attrition, Muskovit, Leukophyllit, Simulation

Modernisation of the Wet Processing Unit in Aspang

Abstract: The wet processing unit in Aspang is used for the production of muscovite mica out of leucophyllite from the open pit in Aspang. The modernisation had the aim to increase the output of muscovite mica and to improve the energy efficency. The increase of the muscovite mica output is caused due to the installation of an attrition machine. In addition, the wet processing plant was adapted for the production of washed quartz sand for the concrete industry. For this purpose the overlaying "Semmering quarzites" are used.

Keywords: Modernisation, Aspanger, Attrition, Muscovite, Leucophyllite, Simulation

\section{Einleitung}

Die Aspanger Bergbau und Mineralwerke GmbH \& Co KG betreibt seit 1856 einen Leukophyllitbergbau in Aspang am Wechsel. Dieser sog. Leukophyllit (Abb. 1 und 2), welcher petrographisch richtigerweise als Leukophyllonit zu bezeichnen wäre, besteht im Wesentlichen aus Muskovit und Quarz, wobei der Muskovit das Wertmineral darstellt. Der vorliegende Leukophyllit kann makroskopisch in drei Typen (I-III) eingeteilt werden, wobei der Typ III in zwei Untertypen (IIla und IIIb) gegliedert werden kann. Zudem tritt noch ein dunkler Phyllit (IV) auf, der als Zwischenmittel bezeichnet wird. Dieser Phyllit lässt sich in zwei Untertypen (IVa und IVb) gliedern und wird ausschließlich verhaldet. Im Leukophyllit treten zudem Karbonate - Calcit und Dolomit, sowie Feldspäte - Albit und Orthoklas auf. Lediglich im Leukophyllit Typ IIlb kommt Chlorit vor. Als Akzessorien kommen Sulfide vor, vorrangig ist hierbei Pyrit zu nennen.

Aufgrund der Lagerstättengegebenheiten erfolgt die Aufbereitung des im Reißbetrieb gewonnenen Leukophyllits im ersten Schritt in einer nass betriebenen Aufbereitungsanlage. In dieser Aufbereitungsanlage, die auch als Nassaufbereitung bezeichnet wird, erfolgt die Trennung des Leukophyllits auf Basis der Korngröße in vier Teilströme: Muskovit, Schlicker, Sandfraktion und grobe Gesteinskörnung (Abb. 1).

Das Leukophyllitflöz wird von Semmeringquarziten überlagert. Diese Quarzite, die hinsichtlich des Gefüges als metamorphe Quarzsandsteine anzusprechen sind, wurden bis dato keiner wirtschaftlichen Verwertung zugeführt, sondern nur zu Rekultivierungszwecken benutzt. Auf Grund der Abbauverhältnisse, d.h. einem Abraum- (=Semmeringquarzit) zu Leukophyllit-Verhältnis von ca. 5:1, muss den Semmeringquarziten eine große wirtschaftliche Bedeutung beigemessen werden. Eine Verwertung dieser Quarzite stellt daher zentrales Thema in der Verwertung dar. 


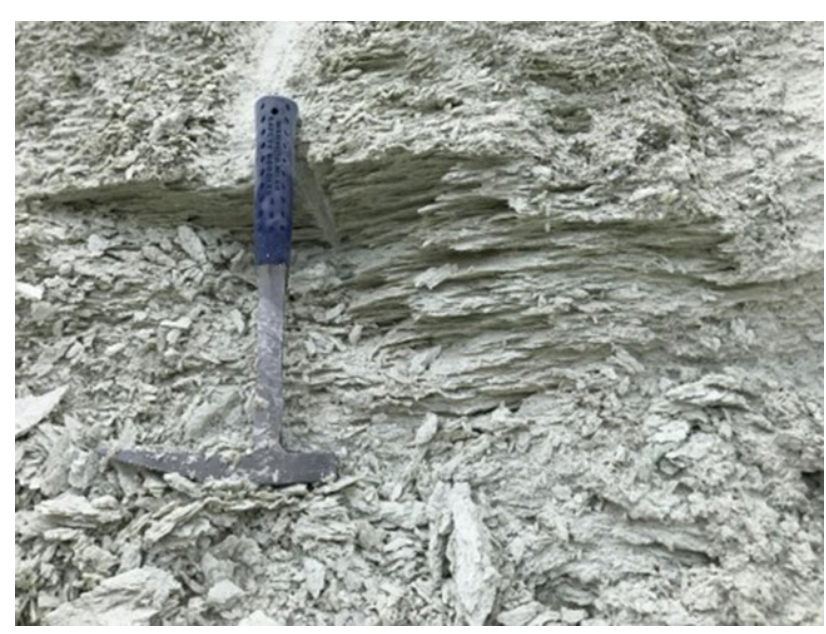

Abb. 1: Leukophyllit

\section{Zielsetzung}

Als Zielsetzung der Modernisierung können zwei Hauptgründe genannt werden. Erstens ist die Erhöhung des Ausbringens an Muskovit zu nennen. Diese Steigerung des Ausbringens erfolgt durch die Reduktion des Muskovitgehalts in der Sandfraktion. Zur Realisierung dieses Vorhabens wurde der Einbau einer 2-zelligen Attritionsmaschine geplant. Die Auslegung der Attrition erfolgte auf Basis von Versuchen, die im Technikum bei Binder+Co in Gleisdorf durchgeführt wurden. Der zweite Aspekt im Zuge der Modernisierung lag in der Nutzung der Semmeringquarzite zur Produktion von gewaschenem Quarzsand für die Betonindustrie. Hierfür wurden im Vorfeld in einer Diplomarbeit [1] die Semmeringquarzite mineralogisch und petrographisch charakterisiert sowie der Vergleich mit anderen Abbaustätten durchgeführt. Auf Basis dieser Erkenntnisse erfolgte die Konzeption der neuen Nassaufbereitungsanlage für die beiden Nutzungsarten. Das Anlagenkonzept der neuen Nassaufbereitung basiert hierbei auf einer Muskovitmenge von ca. 10.000 t und ca. 100.000t Quarzsand pro Jahr.

\section{Ausgangssituation}

Die bestehende Nassaufbereitungsanlage stammt in ihren Grundzügen aus den fünfziger Jahren. In dieser Ära wurde der mittels Nassaufbereitung gewonnene Muskovit-Glimmer ausschließlich als Massenfüllstoff in die Papierindustrie geliefert. Hierbei ist anzumerken, dass es nach der Trennung in den Hydrozyklonstufen und der anschließenden Entwässerung keine weitere Aufbereitung gab. D.h., der so gewonnene Muskovit-Glimmer gelangte unvermahlen mit einer Nennkorngröße von 0/20 $\mu$ m an die Kunden. 1982 wurde die bestehende Anlage letztmalig adaptiert, im Zuge dieses Umbaus wurde einerseits der $66^{\prime \prime}$-Schraubenklassierer als auch das derzeit im Einsatz befindliche Hydrozyklonsystem in die Anlage integriert. Die Auslegung der Nassaufbereitungsanlage basierte auf einer Produktions-

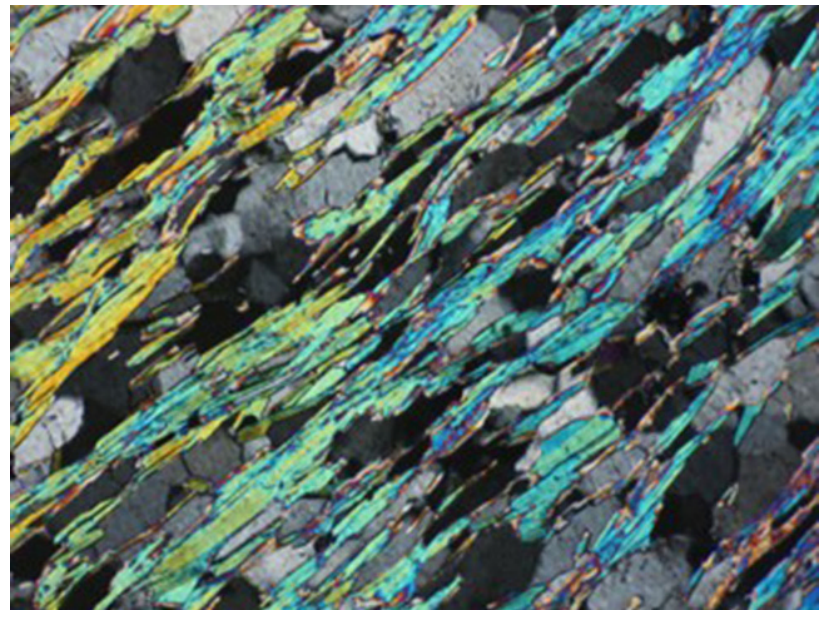

Abb. 2: Mikroskopieaufnahme des Leukphyllits, Bildbreite entspricht ca. $4 \mathrm{~mm}$

menge von ca. 70.000 t Muskovit pro Jahr. Das Konzept der Nassaufbereitung kann wie folgt beschrieben werden. Die Aufgabe des Leukophyllits in die Nassaufbereitung erfolgt mittels Radlader. Das Aufgabegut hat hierbei eine maximale Korngröße von ca. $250 \mathrm{~mm}$. Die größeren Gesteinsbrocken des Rohgutes werden über einen Rost abgeschieden. Anschließend wird der Leukophyllit mit Hilfe einer Prallmühle auf eine Nennkörnung $0 / 40 \mathrm{~mm}$ gebrochen und mittels Förderband der Läutertrommel zugeführt. In der Läutertrommel wird der Leukophyllit mit Wasser aufgeschlämmt. Die ungefähre Wassermenge, die für diesen Schlämmprozess benötigt wird, kann mit ca. $650 \mathrm{~m}^{3} / \mathrm{h}$ angegeben werden. Um eine zusätzliche Zerkleinerungswirkung in der Läutertrommel zu erreichen, wird diese mit Mahlkörpern beaufschlagt. Die so zerkleinerte und aufgeschlämmte Leukophyllitsuspension wird nun mittels des $66^{\prime \prime}$-Schraubenklassierers in die sog. Trübe und in eine Grobfraktion getrennt. Der Trennschnitt des Schraubenklassierers beträgt ca. $100 \mu \mathrm{m}$. Die Grobfraktion wurde anschließend auf einer Eindecker-Kreisschwinger-Siebmaschine in die Sandfraktion 0/4 mm und den Siebüberlauf der Nennkörnung $4 / 40 \mathrm{~mm}$ getrennt. Diese beiden Fraktionen wurden nur untergeordneten Verwendungszwecken zugeführt. Die Überlauftrübe des Schraubenklassierers wird in einem 3-stufi-

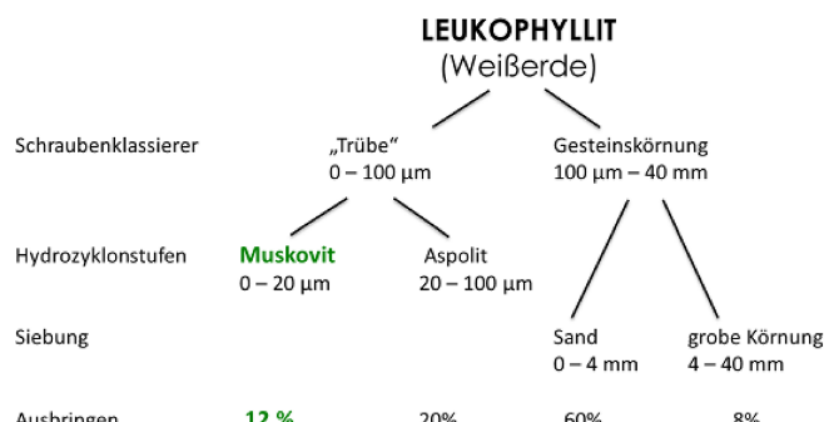

Abb. 3: Teilströme der Leukophyllitaufbereitung 
gen Hydrozyklonsystem weiter aufgetrennt. Diese Fraktionierung führt zu drei Teilströmen (Abb. 3). Die beiden feinen Teilströme mit einem Kornband von 0/15 $\mu \mathrm{m}$ bzw. 0/20 $\mu \mathrm{m}$ sind Muskovitprodukte mit Muskovitgehalten des Feststoffes von ca. 95\%. Der dritte, gröbere Teilstrom aus der Trennung der Trübe weist ein Korngrößenband von ca. 20 bis $100 \mu \mathrm{m}$ auf und ist hinsichtlich der mineralogischen Zusammensetzung als Mischung aus Muskovit und Quarz zu bezeichnen. Untergeordnet treten Feldspäte und Karbonate auf. Dieser Teilstrom wird auch als Schlicker bezeichnet.

\section{Konzept der neuen Nassaufbereitungs- anlage}

\subsection{Muskovitproduktion}

Das Konzept der neuen Nassaufbereitung sieht neben der Optimierung der Muskovitproduktion auch die Produktion von Quarzsand für die Betonindustrie vor. Die Optimierung der Muskovitproduktion ist im ersten Schritt durch die Erhöhung des Ausbringens an Muskovit bedingt, ohne Veränderung der Trennschnitte in den Hydrozyklonstufen, die in der ersten Modernisierungsphase unberührt bleiben. Da der Trennschnitt im $66^{\prime \prime}$-Schraubenklassierer unverändert bleibt, kann die Optimierung nur durch eine Verringerung des Muskovitgehalts in der Sandfraktion erfolgen. Aus mineralogischen Untersuchungen konnte ein Muskovitgehalt in der Sandfraktion 0/4 mm von ca. 20 bis $30 \%$ ermittelt werden. Der Muskovit, der in der Sandfraktion enthalten ist, ist einerseits in Körnern gebunden, andererseits an gröberen Körnern anhaftend (Abb. 4). Dieser anhaftende Anteil soll durch den Einsatz einer Attritionsmaschine dem Trübestrom zugeführt werden, um so das Ausbringen zu erhöhen.

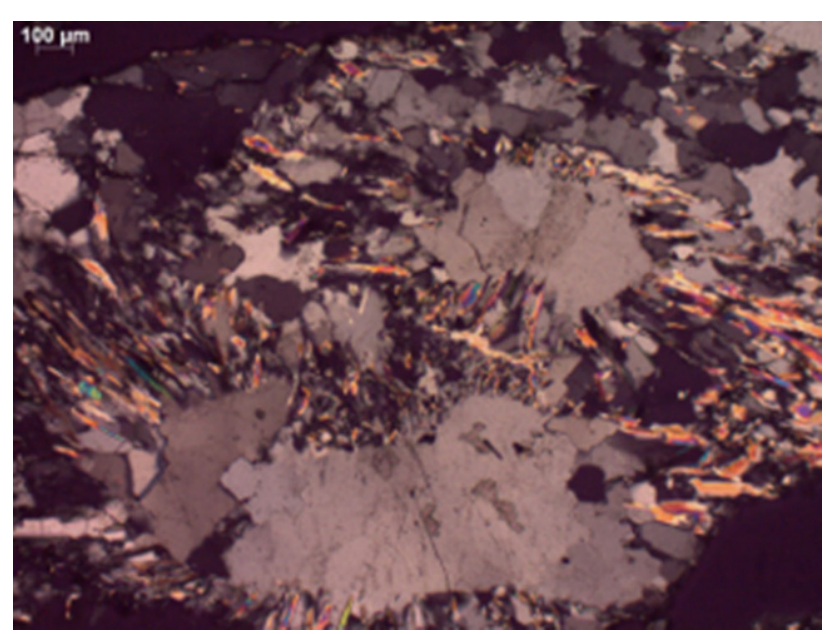

Abb. 4: Mikroskopieaufnahme der Sandfraktion ohne Attrition, Bildbreite entspricht ca. $4 \mathrm{~mm}$

Um die Machbarkeit im Vorfeld zu testen, wurden im Technikum der Binder+Co AG in Gleisdorf Versuche mit einer 2-zelligen Attritionsmaschine durchgeführt. Diese Versuche zeigten die Möglichkeit einer deutlichen Erhöhung des Muskovitausbringens und bildeten die Grundlage für die Auslegung der Attritionsmaschine sowie der gesamten Nassaufbereitung.

Die Modernisierung der Nassaufbereitung wurde an Hand des Konzepts von Binder+Co beginnend mit Oktober 2016 umgesetzt (Abb. 5).

Die neuen aufbereitungstechnischen Kernkomponenten des Konzepts waren eine Kreisschwinger-Siebmaschine mit Bebrausungsanlage (Sieb 1) für einen nominellen Trennschnitt von $8 \mathrm{~mm}$ oder $4 \mathrm{~mm}$, ein Schraubenklassierer für einen nominellen Trennschnitt von $160 \mu \mathrm{m}$, eine Attritionsmaschine zur Läuterung des Schraubenklassie-
Abb. 5: Vereinfachtes Verfahrensschema der neuen Nassaufbereitung-Muskovitproduktion

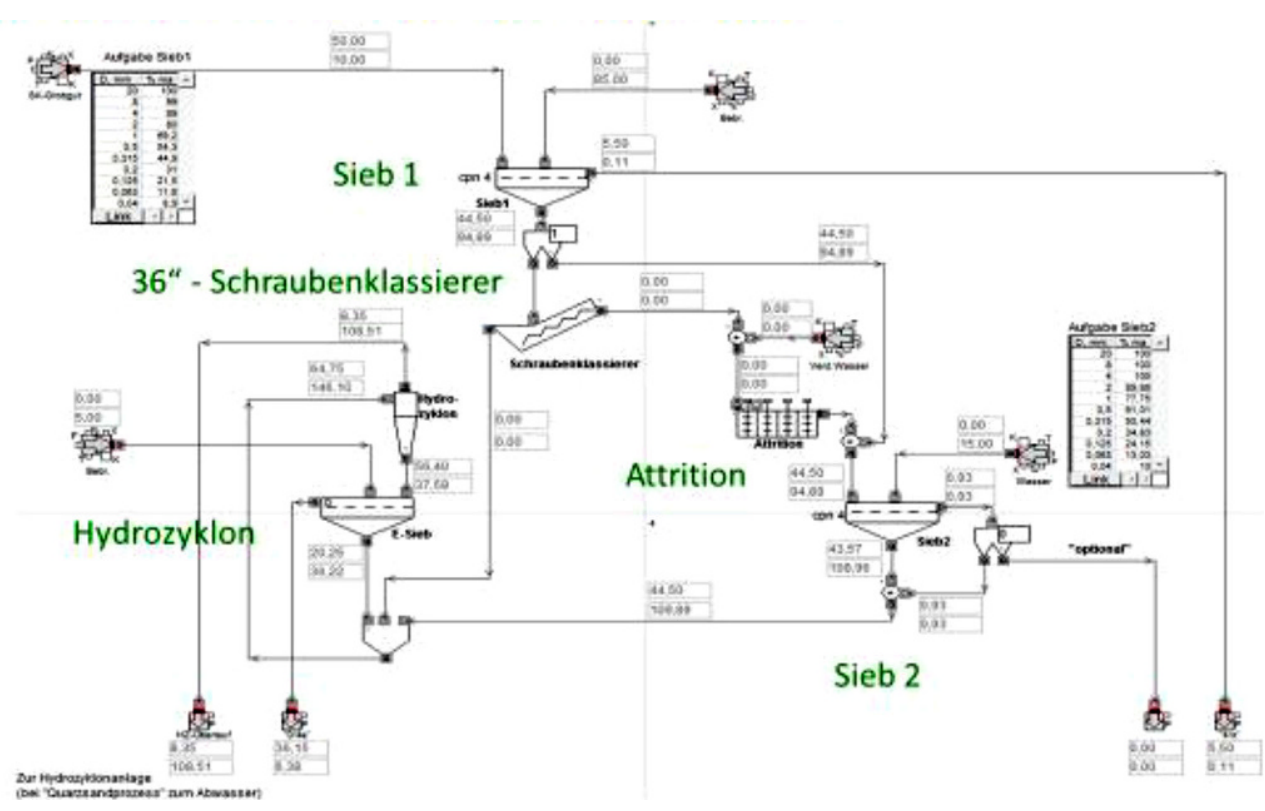


rer-Grobgutes, ein weiterer Kreisschwinger mit Bebrausungsanlage (Sieb 2) zur optionalen Absiebung eines Produktes $4 / 8 \mathrm{~mm}$, ein Hydrozyklon zur Abscheidung einer Feinguttrübe und eine Entwässerungssiebmaschine mit Bebrausungsanlage zur Entwässerung sowie zusätzlichen Entschlämmung der Hydrozyklon-Unterlauftrübe $0 / 4 \mathrm{~mm}$. Hinsichtlich aufbereitungstechnischer Maschinenauslegung kamen neben der Berücksichtigung von Versuchsauswertungen ein Simulationsprogramm (vereinfachtes Fließschema siehe Abb. 5 und 8) und diverse Berechnungsprogramme zur Auslegung der einzelnen Maschinen zum Einsatz.

Bei den Simulationsrechnungen erwies sich zur Beschreibung der Stoffströme die Festlegung von einer aus 11 Merkmalsklassen (Korngrößenklassen) bestehenden Feststoffphase und einer Wasserphase als zielführend. Als obere Kornklassengrenzen zu den Korngrößenklassen galten $20 \mathrm{~mm}, 8 \mathrm{~mm}, 4 \mathrm{~mm}, 2 \mathrm{~mm}, 1 \mathrm{~mm}, 0,5 \mathrm{~mm}, 0,315 \mathrm{~mm}$, $0,2 \mathrm{~mm}, 0,125 \mathrm{~mm}, 0,063 \mathrm{~mm}$ und $0,04 \mathrm{~mm}$. Der Aufgabestoffstrom konnte durch die Angabe der FeuchtgutFließrate in $\mathrm{t} / \mathrm{h}$, die Angabe von Feststoffgehalt bzw. Wassergehalt in \% und die Angabe der Durchgangswerte in $\%$ zu den vorhin genannten Korngrößenklassengrenzen beschrieben werden. Zur Simulation von "Sieb 1“ diente ein sehr einfaches Rechenmodell, wobei als wesentliche Rechenmodellparameter der nominelle Trennschnitt und der Wassergehalt des Siebüberlaufs zu nennen sind. Die Brausewassermenge wurde gemäß einem zweiten Aufgabestoffstrom unter Angabe der Fließrate in $\mathrm{t} / \mathrm{h}$ und Angabe eines Wassergehaltes von $100 \%$ berücksichtigt. Das Rechenmodell "Splitter" ermöglicht die Lenkung der Siebunterlauftrübe von "Sieb 1“ zum 36"-Schraubenklassierer oder in Richtung "Sieb 2" (Umgehung der Attrition beim "Quarzsandprozess"; siehe Abschn. 4.2). Die Trenncharakteristik des $36^{\prime \prime}$-Schraubenklassierers basiert gleichsam auf vier Rechenmodellparametern. Drei Parameter dienen der Festlegung der grobgutbezogenen Teilungszahlen zu den einzelnen Korngrößenklassen. Mit dem vierten Parameter wird der Wassergehalt des SchraubenklassiererGrobgutes festgelegt, woraus sich zu jedem Iterationsschritt der jeweiligen Simulationsrechnung automatisch die entsprechenden Fließraten an Wasser sowohl für das Klassierergrobgut als auch für die Klassiererfeinguttrübe errechnen. Vor der Attrition ermöglicht ein dritter Aufgabestoffstrom die optionale Zugabe von Verdünnungswasser. Hinsichtlich der Attrition ist ein sehr einfaches Rechenmodell hinterlegt, das im Wesentlichen nur der Darstellung der Massenflüsse dient. Für die Auslegung der Attritionsmaschine waren vorwiegend die in Gleisdorf durchgeführten Versuche relevant. Zur Simulation von „Sieb 2" diente ein Rechenmodell mit vier Modellparametern. Drei Parameter dienen der Festlegung der grobgutbezogenen Teilungszahlen zu den einzelnen Korngrößenklassen. Mit dem vierten Parameter wird der Wassergehalt des Siebüberlaufs festgelegt, woraus sich zu jedem Iterationsschritt automatisch die entsprechenden Fließraten an Wasser sowohl für den Siebüberlauf als auch für die Siebunterlauftrübe errechnen. Die Brausewassermenge für "Sieb 2" wurde gemäß einem vierten Aufgabestoffstrom in ähnlicher Weise wie bei „Sieb 1“ berücksichtigt. Die Unterlauftrübe von „Sieb 2 ” und die Überlauftrübe des $36^{\prime \prime}$-Schraubenklassierers werden der Hydrozyklon-Kompaktanlage zugeführt.

Die Hydrozyklon-Kompaktanlage entspricht einer zweistufigen Klassierung. Als erste Stufe gilt der eigentliche Zyklon. Als zweite Stufe dient eine Entwässerungssiebmaschine mit Bebrausungsanlage, wobei die Siebunterlauftrübe gemäß einem Kreislaufstrom wiederum dem Hydrozyklon aufgegeben wird. Das Rechenmodell zur Simulation des Hydrozyklons und das Rechenmodell zur Simulation der Entwässerungssiebmaschine sind ähnlich strukturiert wie jenes zur Simulation des Schraubenklassierers. Die Produkte der Hydrozyklon-Kompaktanlage sind der Hydrozyklonüberlauf (Dünntrübe) und der Überlauf der Entwässerungssiebmaschine (entwässertes und „entschlämmtes" Sandprodukt). Bei der Muskovitproduktion wird der Hydrozyklonüberlauf als wertvolles Zwischenprodukt zum 3-stufigen Hydrozyklonsystem ("Altbestand“) weitergeleitet, beim Quarzsandprozess kann der Hydrozyklonüberlauf als Abwasser bezeichnet werden.

Die Simulationsrechnungen lieferten zu den entsprechenden Stoffströmen die Fließraten an Feststoff und Wasser sowie die zugehörigen Korngrößenverteilungen und bildeten somit die Basis zur aufbereitungstechnischen Maschinenauslegung.

Beginnend mit Oktober 2016 wurde der Abriss der alten Anlage in Angriff genommen. Die Modernisierung umfasst die Generalsanierung der Läutertrommel und des $66^{\prime \prime}$-Schraubenklassierers sowie den kompletten Neubau der Aufbereitungsanlagen für die Grobfraktion. Die Hydrozyklonstufen und die damit verbundenen Aggregate sind von dieser ersten Umbauphase nicht betroffen. Beim $66^{\prime \prime}$-Schraubenklassierer ist anzumerken, dass dieser von einem 3-gängigen auf einen 1-gängigen rückgebaut wurde.

Im Anschluss an den 66"-Schraubenklassierer wurde eine Eindecker-Kreisschwinger-Siebmaschine $1 \times 1,3 \mathrm{~m}$ installiert. Mit dieser Maschine wird der Trennschnitt von $4 \mathrm{~mm}$ realisiert (siehe Abb. 5; „Sieb 1“ im Fließschema des Simulationsprogramms). Die Nennkörnung größer $4 \mathrm{~mm}$ wird nicht weiter behandelt und mittels Förderband auf Halde transportiert. Die Siebunterlauftrübe $0 / 4 \mathrm{~mm}$ wird zum neuen $36^{\prime \prime}$-Schraubenklassierer gepumpt. Hier erfolgt die Nachwäsche der Sandfraktion. Die dabei gewonnene Feinguttrübe, die de facto reinen Muskovit beinhaltet,

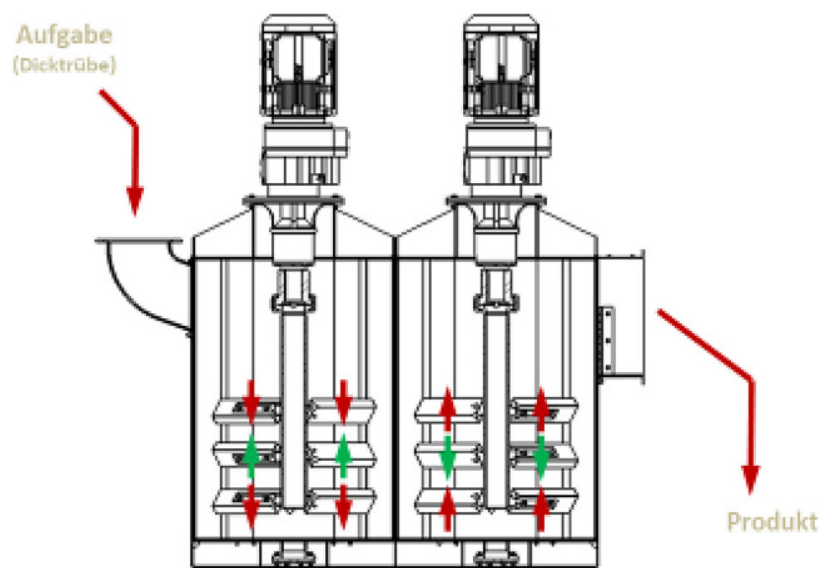

Abb. 6: 2-zellige Attrition 
Abb. 7: Ausbringen an Muskovit im Betrachtungszeitraum 2003-2017

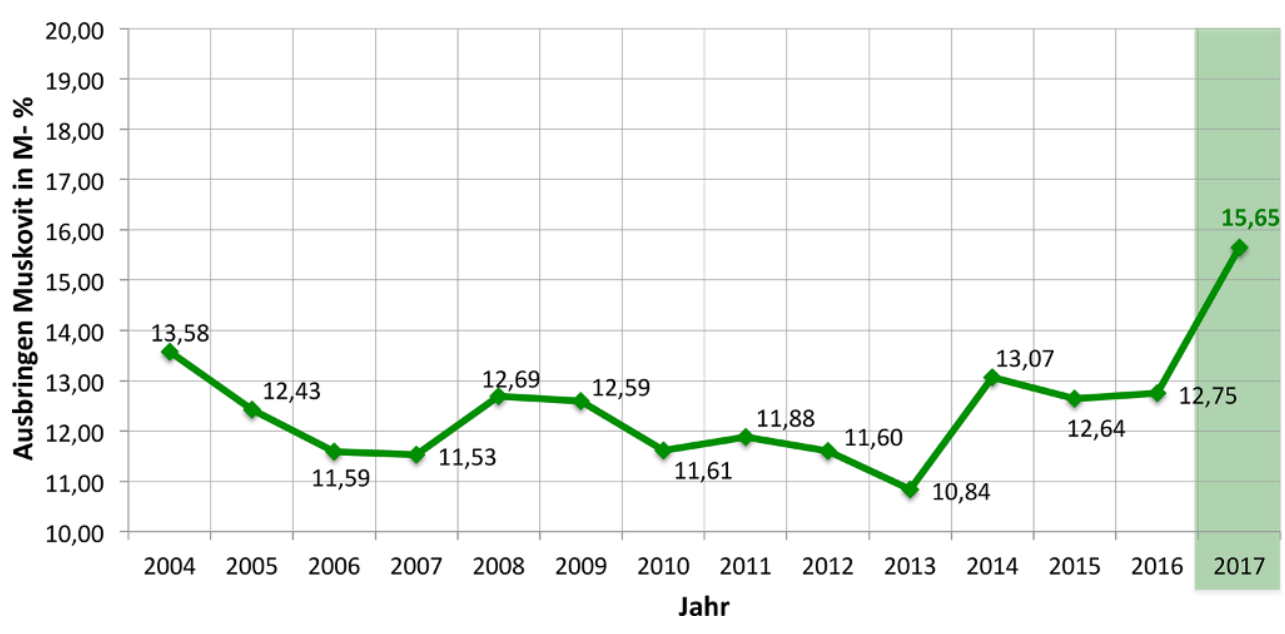

Steigerung des Ausbringens von 12,2\% auf $15,7 \%$ erzielt werden konnte (Abb. 7).

Dies bedeutet, dass um die gleiche Menge Muskovit zu produzieren, der Leukophyllitabbau um ca. $22 \%$ zurückgefahren werden kann, woraus sich neben dem ökonomischen Effekt eine Schonung der Lagerstätte ergibt.

\subsection{Quarzsandproduktion}

Die Quarzsandproduktion stellt den zweiten Betriebszustand der Nassaufbereitung dar. Im Wesentlichen ist die Konzeption der Quarzsandproduktion ähnlich wie jene der Muskovitproduktion. Der Unterschied besteht darin, dass die Überlauftrübe des $66^{\prime \prime}$-Schraubenklassierers in Absetzbecken gepumpt und anschließend verhaldet wird, das Hydrozyklon-System wird in diesem Betriebszustand nicht benötigt. Zusätzlich wird, um den Verschleiß hintanzuhalten, der $36^{\prime \prime}$-Schraubenklassierer und die Attritions-
Abb. 8: Vereinfachtes Verfahrensschema der neuen Nassaufbereitung-Quarzsandproduktion

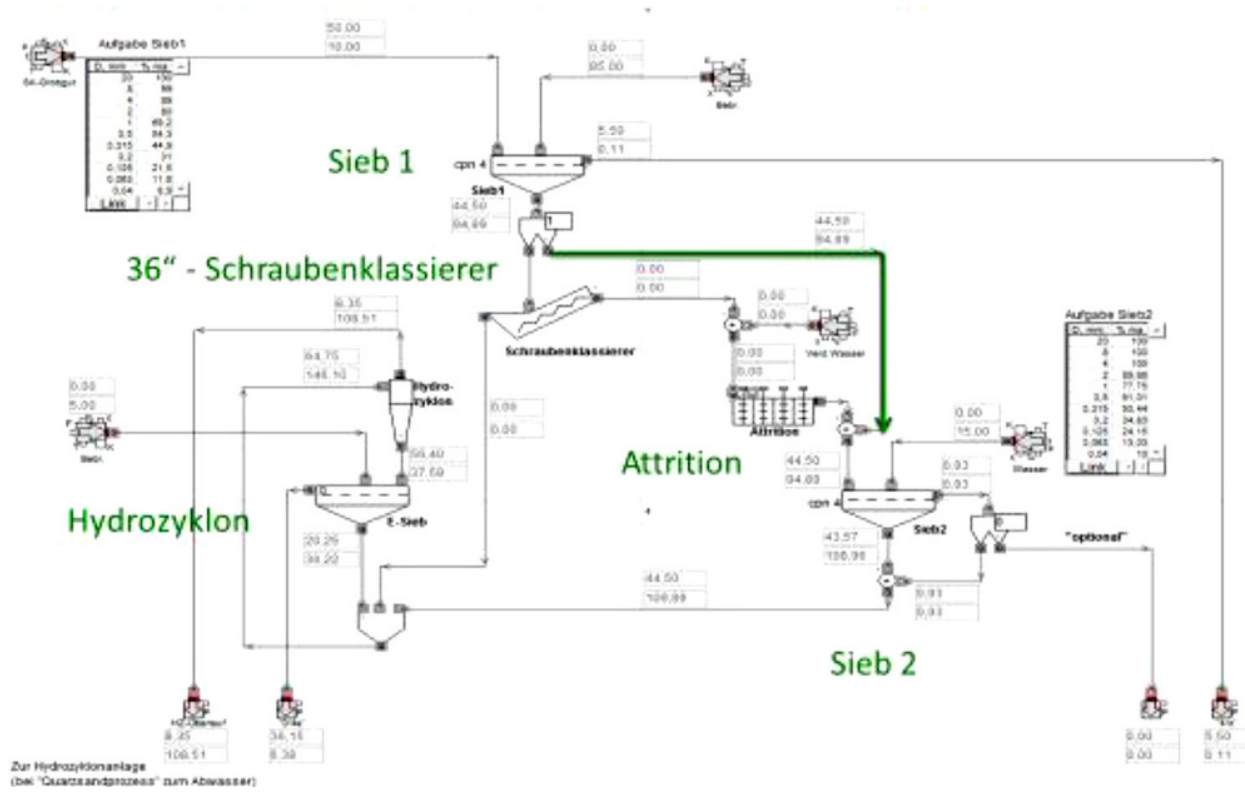


maschine mittels einer Bypasslösung umfahren, sodass der Quarzsand direkt nach der ersten Kreisschwinger-Siebmaschine der Hydrozyklon-Kompaktanlage zugeführt wird (Abb. 8). Um die Verwendbarkeit der in Aspang vorliegenden Semmeringquarzite zu evaluieren, wurde bereits im Jahr 2015 eine Diplomarbeit zur Charakterisierung dieser Quarzite vergeben [1]. Auf Basis der Erkenntnisse dieser Arbeit wurden die Vorversuche und anschließend die Erstprüfung gemäß der ÖNORM EN 12620 - Gesteinskörnungen für Beton [2] vorgenommen. Mit der Durchführung der Erstprüfung wurde die Materialprüfanstalt Hartl $\mathrm{GmbH}$ [3] beauftragt. Hierbei ist anzumerken, dass der Sand hinsichtlich des Gehaltes an Feinanteilen der Kategorie f3 [2] entspricht, somit für den Einsatz für Spritzbeton gemäß OVBB-Richtlinie Spritzbeton [4] prinzipiell geeignet ist. Ein wichtiger Punkt für den Einsatz in der Betonindustrie stellt zudem die Beurteilung der Alkali-Silika-Reaktivität dar. Diese Beurteilung erfolgt gemäß den Anforderungen der ÖNORM B 3100 [5], wobei zwei Möglichkeiten der Beurteilung geben sind. Einerseits der petrographische Vergleich innerhalb eines Abbaugebietes [6], für welches positive Erfahrungen hinsichtlich der AKR-Beständigkeit vorliegen, andererseits die Prüfung der AKR im Schnell- oder Langzeittest $[7,8]$. In diesen Tests wird die Längenänderung eines Mörtel- bzw. Betonkörpers nach Lagerung in einer einmolaren $\mathrm{NaOH}$-Lösung beurteilt. Beide Tests wurden positiv bestanden, sodass den Anforderungen für einen Einsatz in der Betonindustrie grundlegend genüge getan wurde. Im Gegensatz zur Produktion von Muskovit wurde die Anlage bei dieser Fahrweise auf eine Aufgaberate von $50 \mathrm{t} / \mathrm{h}$ ausgelegt, wobei die Voruntersuchungen ein Ausbringen an Quarzsand von ca. $70 \%$ ergaben. Zu erwähnen ist, dass neben den Feinanteilen $(<0,063 \mathrm{~mm})$ noch eine Grobfraktion analog der Muskovitproduktion an der ersten Siebmaschine abgetrennt wird. Diese Körnung weist eine Nennkorngröße von 4/32 mm auf und fällt mit einem Masseausbringen von ca. $20 \%$ an. Aufgrund des vorliegenden Sandsteingefüges der Semmeringquarzite weist diese grobe Gesteinskörnung niedrige Kornfestigkeit auf, was sich in hohen Los Angeles-Koeffizienten gemäß ÖNORM EN 1097-2 widerspiegelt $[9,10]$. Aus diesem Grund erfolgt die normative Einstufung gemäß der ÖNORM EN 13242 [11] für untergeordnete Anwendungen. Auf Basis der ersten Produktionsmonate konnte ein Ausbringen an Quarzsand von ca. $65 \%$ ermittelt werden, wobei die nächste Zielsetzung eine Steigerung des Sandausbringens und somit eine Verringerung des Anteils der groben Gesteinskörnung ist.

Funding. Open access funding provided by Montanuniversität Leoben.

Open Access Dieser Artikel wird unter der Creative Commons Namensnennung 4.0 International Lizenz (http://creativecommons.org/licenses/ by/4.0/deed.de) veröffentlicht, welche die Nutzung, Vervielfältigung, Bearbeitung, Verbreitung und Wiedergabe in jeglichem Medium und Format erlaubt, sofern Sie den/die ursprünglichen Autor(en) und die Quelle ordnungsgemäß nennen, einen Linkzur Creative Commons Lizenz beifügen und angeben, ob Änderungen vorgenommen wurden.

\section{Literatur}

1. Reiß, S.: Mineralogische und petrographische Charakterisierung der Semmeringquarzite mit dem Hauptaugenmerk des Einsatzes in zementär gebundenen Baustoffen, Masterarb., Leoben, Montanuniversität, Lehrstuhl für Rohstoffmineralogie, 2016

2. ÖNORM EN 12620 - Gesteinskörnungen für Beton, Ausgabe 15.02.2014

3. Materialprüfanstalt Hartl $\mathrm{GmbH}$ : Prüfbericht über die Überprüfung der natürlichen Gesteinskörnung 0/4 KK, Erstprüfung gemäß ÖNORM EN 12620 und ÖNORM B 3131, Prüfbericht Nr. 4 - 31027 firmeninterner Bericht, 2015

4. Richtlinie Spritzbeton, Dezember 2009, Österreichische Vereinigung für Beton- und Bautechnik

5. ÖNORM B 3100 - Beurteilung der Alkali-Kieselsäure-Reaktivität im Beton, Ausgabe 01.08.2008

6. Thalhammer, O.: Vergleichende mineralogische und petrographische Charakterisierung der Gesteinsproben Asp6 (Aspang) und Fei3 (Haßbach), firmeninterner Bericht, 2015

7. VBE Verein für Baustoffprüfung und -entwicklung, Staatlich akkreditierte Prüf- und Inspektionsstelle : Bestimmung der Alkali-Kieselsäure-Reaktivität, Schnelltest - Prüfbericht Nr. 11464/1, firmeninterner Bericht, 2015

8. VBE Verein für Baustoffprüfung und -entwicklung, Staatlich akkreditierte Prüf- und Inspektionsstelle: Bestimmung der Alkali-Kieselsäure-Reaktivität, Schnelltest - Prüfbericht Nr. 11464/2, firmeninterner Bericht, 2016

9. ÖNORM EN 1097-2 - Prüfverfahren für mechanische und physikalische Eigenschaften von Gesteinskörnungen - Teil 2: Verfahren zur Bestimmung des Widerstandes gegen Zertrümmerung, Ausgabe 01.06.2010

10. Straßentechnologische Prüfanstalt Dipl.-Ing. Vladimir Vasiljevic GesmbH: Prüfbericht AK 3889917 - Widerstand gegen Zertrümmerung, 2017

11. ÖNORM EN 13242 - Gesteinskörnungen für ungebundene und hydraulisch gebundene Gemische für Ingenieur- und Straßenbau, Ausgabe 15.02.2014 